\title{
Togitare
}

\section{HÁBITOS DE VIDA DE PESSOAS COM DIABETES MELLITUS DURANTE A PANDEMIA DE COVID-19}

\author{
Giulia Gabriella de Oliveira Pedroza ${ }^{1}$ (1) \\ Anne Caroline de Moraes Monção ${ }^{1}$ (1) \\ Heitor de Oliveira Valladares ${ }^{1}$ (i) \\ Sávio Dias de Paula Mello ${ }^{1}$ (1) \\ Victor Hugo de Maria Paura de Souza ${ }^{1}$ (1) \\ Júlio Cesar Santos da Silva ${ }^{1}$ (i) \\ Marcela dos Santos Ferreira ${ }^{1}$ (1)
}

\begin{abstract}
RESUMO
Objetivo: caracterizar impactos do distanciamento social ocasionado pela pandemia da doença do Coronavírus na vida de pessoas com Diabetes Mellitus.

Método: estudo descritivo quantitativo, realizado com 102 portadores de Diabetes Mellitus, por meio de formulário virtual, abordando questões sociodemográficas, de autocuidado e de saúde mental, no Brasil no período de maio a junho de 2020. A associação entre as variáveis foi avaliada pelo teste qui-quadrado $(p<0,05)$.

Resultados: dos 45 (44,11\%) que disseram se alimentar mal, 33 (32,35\%) relataram mudanças de peso $(p=0,008)$; com relação à saúde mental, 59 (57,84\%) expuseram condições regulares ou ruins; com relação às consultas, 84 indivíduos (82,75\%) alegaram interrupção ou irregularidade; dos 58 $(56,86 \%)$ que declararam prejuízos socioeconômicos, 37 (36,27\%) apresentaram dificuldades na adesão de insumos $(p<0,000)$.

Conclusão: medidasmultiprofissionais devem serfocadas nos fatores que influenciam negativamente o controle metabólico, assim como em estabelecer atendimento a distância, para minimizar a diminuição da atenção assistencial.
\end{abstract}

DESCRITORES: Diabetes Mellitus; Infecções por Coronavírus; Pandemia; Hábitos; Isolamento Social.

\section{HÁBITOS DE VIDA DE PERSONAS CON DIABETES MELLITUS DURANTE LA PANDEMIA DE COVID-19}

\section{RESUMEN:}

Objetivo: caracterizar los impactos del distanciamiento social provocado por la pandemia de la enfermedad por Coronavirus en la vida de las personas con Diabetes Mellitus. Método: estudio descriptivo cuantitativo, realizado con 102 pacientes con Diabetes Mellitus, a través de un formulario virtual, con el abordaje de temas sociodemográficos, de autocuidado y de salud mental, en Brasil de mayo a junio de 2020. La asociación entre variables fue evaluada por la prueba de chi-cuadrado ( $p<0,05)$. Resultados: de los $45(44,11 \%)$ que admitieron comer mal, 33 (32,35\%) reportaron cambios de peso ( $p=0,008)$; con respecto a la salud mental, $59(57,84 \%)$ expusieron condiciones regulares o malas; En cuanto a las consultas, 84 personas (82,75\%) alegaron interrupción o irregularidad; de los 58 (56,86\%) que declararon pérdidas socioeconómicas, 37 (36,27\%) tuvieron dificultades en la adhesión de insumos ( $p<0,000)$. Conclusión: las medidas multiprofesionales deben centrarse en los factores que influyen negativamente en el control metabólico, así como en el establecimiento de cuidados a distancia, para minimizar la disminución de la atención asistencial.

DESCRIPTORES: Diabetes Mellitus; Infecciones por Coronavirus; Pandemia; Hábitos; Aislamiento Social. 
O novo coronavírus (SARS-CoV-2) é um vírus recém-emergido e altamente contagioso, causador da doença do Coronavírus (covid-19), que invade o trato respiratório e os pulmões, levando a um novo tipo de pneumonia por coronavírus ${ }^{(1)}$. Segundo a Organização Pan-americana de Saúde, somente na região das Américas o número de casos confirmados, em julho de 2020, já ultrapassava 3.868.569 milhões e no Brasil 2.292.286 milhões. Mundialmente, esse número ultrapassa 15.012.731 milhões de acometidos pela doença. A Organização Mundial da Saúde (OMS) divulgou que o número de óbitos era de $3,4 \%$ do total de infectados, caracterizando a seriedade da pandemia que o mundo está enfrentando(1).

Há um grupo de pessoas que é considerado mais vulnerável a doença causada pelo SARS-CoV-2, composto por idosos e com pessoas que possuem condições pré-existentes, como hipertensão, câncer, doenças cardiovasculares, Diabetes Mellitus (DM) ${ }^{(1)}$. Tais comorbidades aumentam o risco de desenvolver casos mais graves de covid-19, além de maior risco de mortalidade ${ }^{(2-3)}$. Sabe-se que dos casos da doença, $81 \%$ serão leves, $14 \%$ graves e $5 \%$ críticos $^{(4)}$.

Muitos indivíduos, ao terem contato com o novo coronavírus, poderão sentir sintomas leves da doença ou até mesmo serem assintomáticas, no entanto, portadores de DM podem precisar de internação hospitalar com maior frequência para receber cuidados e intervenção intensiva(4). Embora estudos demonstrem números distintos acerca da letalidade e morbidade dos pacientes com covid-19 e comorbidades, todos eles demonstram que pessoas deste grupo têm maior probabilidade a evoluir ao estado grave e crítico da doença.

Em um estudo com 138 pacientes, verificou-se que $72 \%$ dos pacientes com COVID-19 e comorbidades, incluindo DM, necessitaram de cuidados intensivos, comparado a $37 \%$ dos pacientes sem comorbidades ${ }^{(5)}$. Outro estudo, que incluiu 1099 pacientes na China, observou que dentre os 173 com os sintomas em nível grave da covid-19, 23,7\% apresentavam hipertensão, 16,2\% DM, 5,8\% doenças cardíacas e 2,3\% doença cerebrovascular ${ }^{(6)}$. E em um terceiro estudo chinês, com 140 pacientes internados no hospital com covid-19, 30\% tinham hipertensão e $12 \%$ tinham $\mathrm{DM}^{(7)}$.

Segundo a Sociedade Brasileira de Diabetes (SBD), em consonância com as informações do Ministério da Saúde (MS), os portadores de DM pertencem ao grupo de risco da covid-19 devido à baixa imunidade, ligada à elevação do açúcar no sangue. Entretanto, a SBD informa que portadores de DM que possuem controle da glicemia por intermédio da monitoração, uso adequado de insulina ou medicação oral, alimentação equilibrada e atividade física, tem maior facilidade de enfrentar a covid-19, ou seja, há menor risco para casos graves e críticos da doença( ${ }^{(8)}$.

No Brasil, devido ao alto risco da covid-19, os portadores de DM (entre outras doenças crônicas), idosos e gestantes devem se manter em distanciamento social, baseado em recomendação do MS (Portaria n. 65/2020) ${ }^{(9)}$, incluindo a possibilidade do afastamento de seu campo de trabalho, favorecendo o cumprimento do distanciamento social como medida de prevenção da transmissão. Tal medida ressalta a importância dos portadores de DM respeitarem as recomendações da SBD, MS e OMS na prevenção ao SARS-CoV-2.

Levando em consideração que as mudanças decorrentes desta pandemia tem o potencial de impactar diretamente a vida de um portador de DM, resolveu-se pesquisar acerca da sua rotina, durante este período, com o objetivo de caracterizar impactos do distanciamento social ocasionado pela pandemia da doença do coronavírus na vida de pessoas com DM. 
Trata-se de um estudo descritivo de abordagem quantitativa, realizado com pessoas com DM maiores de 18 anos, sendo o único critério de inclusão. A pesquisa ocorreu durante os meses de maio e junho de 2020, correspondendo parte do período de distanciamento social em decorrência da pandemia de covid-19. A população estudada foi de indivíduos com DM integrantes de uma mídia social, de abrangência nacional, denominada Insulina sem Fronteiras. A escolha desta rede social para a coleta de dados se deu por ela integrar o projeto de extensão que deu origem a esta pesquisa e ser composta por pessoas elegíveis para o estudo.

Os participantes do estudo foram convidados a participar voluntariamente da pesquisa por intermédio de uma postagem na mídia social Insulina sem Fronteiras, hospedada no Instagram $®$ e Facebook $®$. Por meio da postagem da pesquisa em tais redes, os indivíduos tiveram acesso ao instrumento de coleta de dados. Por conta da necessidade da manutenção do distanciamento social, a aproximação e a coleta de dados foram realizadas virtualmente, mas respeitando todos os preceitos éticos da pesquisa com seres humanos.

A amostragem foi não probabilística e por conveniência e cabe destacar que, pelo convite à pesquisa ter sido coletivo, não se tem dados sobre recusa na participação, e o tamanho da amostra foi alcançado automaticamente com o encerramento do tempo estipulado pelos pesquisadores para a coleta de dados.

A coleta de dados foi realizada por meio da ferramenta Formulários do Google através da digitação online dos dados, com questões objetivas e fechadas, que constituíam as variáveis do estudo. O formulário foi composto por 20 questões, contemplando a dimensão sociodemográfica (sexo, idade e renda), dimensão relacionada ao tratamento (monitorização da glicemia capilar, uso de medicações, regularidade de consultas e adesão de insumos) e dimensão dos hábitos de vida (alimentação, atividade física e atividades complementares para o equilíbrio emocional) baseadas no Questionário de Atividades de Autocuidado com o Diabetes, versão traduzida, adaptada e validada para a cultura brasileira $(\mathrm{QAD})^{(10)}$. Além da questão das condições de saúde mental relacionada ao distanciamento social. Todas as respostas foram autorrelatos.

O questionário, disponível por dois meses nas mídias sociais, era acessado por meio de um link, assim como as informações de padrões éticos da pesquisa, e as respostas foram agrupadas automaticamente num banco de dados e posteriormente organizadas em arquivo Excel para categorização e tabulação.

A análise estatística, que ocorreu após o tempo estabelecido para a coleta de dados, compreendeu a análise descritiva, mediante a distribuição das frequências relativas e absolutas das variáveis. Foi realizado o teste Qui-quadrado para as análises inferenciais das associações entre as seguintes variáveis, desfecho e independentes, respectivamente: aumento de peso x alimentação; aumento de peso $x$ atividade física; condição de saúde mental $x$ prejuízo socioeconômico; condição de saúde mental $x$ notícias referentes à pandemia; condição de saúde mental $x$ realização de atividades complementares para o equilíbrio do estado emocional; adesão de insumos $\mathrm{x}$ prejuízos socioeconômicos, adesão de insumos x número de verificações diárias da glicemia capilar.

Para todas as análises inferenciais, foram respeitados os seguintes pressupostos do teste de Qui-quadrado: os dados são selecionados aleatoriamente; todas as frequências esperadas são maiores ou iguais a 1 e não mais de $20 \%$ das frequências esperadas são inferiores a 5. Para as variáveis analisadas pelo teste de Qui-quadrado em que a frequência em uma das classes foi menor que 1, foi utilizada a correção de Yates. Para todas as análises, considerou-se o nível de significância estatística de $p<0,05$. Os valores dos dados apresentados na etapa discussão são arredondamentos dos descritos na etapa resultados. 
O estudo foi aprovado em Comitê de Ética em Pesquisa, sob parecer n. 1.884.837 de 31/04/2017, seguindo padrões éticos contidos na resolução 466/2012 do Conselho Nacional de Saúde. Antes de iniciar a coleta de dados com o questionário virtual, foi inserido um Termo de Consentimento Livre e Esclarecido (TCLE) virtual, composto por uma página de esclarecimento sobre a pesquisa, além da solicitação de autorização para o uso dos dados.

\section{RESULTADOS}

Participaram do estudo 102 pessoas com DM, com as seguintes características: 75 indivíduos diagnosticados com DM do tipo II (73,53\%), 50 pessoas do sexo feminino $(49,02 \%)$ e idade média da amostra de 41,8 anos. Em relação às características da patologia, especialmente relacionadas ao uso da insulina, tem-se: dos portadores do tipo II, 35 não utilizam insulina (34,31\%), 25 utilizam a seringa como instrumento de administração de insulina (24,51\%) e dos 38 indivíduos que utilizam a caneta de insulina $(37,25 \%), 21$ são portadores do tipo I $(20,59 \%)$.

Com o estabelecimento do distanciamento social, o estudo se propôs a investigar a presença de aumento de peso nos indivíduos portadores da DM, correlacionando essa variável ao tipo de alimentação e também à prática recorrente de exercícios físicos. $\mathrm{Na}$ ausência da medição do peso, seja pela falta de contato com os sujeitos de estudo ou pela dificuldade de acessar uma balança pelo distanciamento social, foi considerada a autopercepção dos entrevistados em relação ao seu peso. A Tabela 1 evidencia a associação do aumento de peso à má alimentação $(p=0,008)$ e também ao déficit de exercícios físicos $(p=0,03)$.

Tabela 1 - Influência da alimentação e da prática de atividades físicas no aumento de peso autopercebido dos portadores de diabetes mellitus, no período de distanciamento social. Rio de Janeiro, RJ, Brasil, 2020

\begin{tabular}{|c|c|c|c|c|c|}
\hline \multirow[t]{2}{*}{ Variáveis } & \multicolumn{2}{|c|}{$\begin{array}{c}\text { Sofreram aumento de } \\
\text { peso }\end{array}$} & \multicolumn{2}{|c|}{$\begin{array}{c}\text { Não sofreram mudanças de } \\
\text { peso }\end{array}$} & \multirow[t]{2}{*}{$\mathbf{p}$} \\
\hline & $\mathbf{n}$ & $\%$ & $\mathbf{n}$ & $\%$ & \\
\hline \multicolumn{6}{|c|}{ Alimentação saudável } \\
\hline Sim & 27 & 26,47 & 30 & 29,41 & \multirow[t]{2}{*}{$0,008^{\star *}$} \\
\hline Não & 33 & 32,35 & 12 & 11,76 & \\
\hline \multicolumn{6}{|c|}{ Prática de Atividade física } \\
\hline Sim & 7 & 6,86 & 12 & 11,76 & \multirow[t]{2}{*}{$0,030^{\star \star}$} \\
\hline Não & 53 & 51,96 & 30 & 29,41 & \\
\hline
\end{tabular}

Outro aspecto estudado foi a qualidade que os entrevistados classificaram a sua saúde mental, tendo em vista que o período de distanciamento social poderia afetá-la, 
principalmente como consequência das notícias referentes à pandemia e em alguns casos com os prejuízos socioeconômicos que ela trouxe à renda de alguns desses indivíduos.

A Tabela 2 apresenta a associação da qualidade da saúde mental com os prejuízos socioeconômicos $(p=0,011)$, ao fato de observar as notícias referentes à pandemia $(p=0,001)$ e ao ato de realizar atividades complementares com o intuito de estabelecer o equilíbrio emocional $(p=0,000)$. Com relação a essas práticas, $22(21,56 \%)$ relataram realizar trabalhos manuais (cuidados com a casa, cozinhar, artesanatos, confecção de hortas), cinco (5,88\%) relataram utilizar esse tempo para estudar e ler, e $17(16,67 \%)$ realizam atividades de lazer (ouvir música, jogar videogame, brincar com o irmão(ã) e 55 (55,89\%) não implementaram nenhuma atividade.

Tabela 2 - Condições da saúde mental em relação à diminuição de renda, notícias referentes à pandemia e realização de atividades complementares. Rio de Janeiro, RJ, Brasil, 2020

\begin{tabular}{|c|c|c|c|c|c|c|c|}
\hline \multirow[t]{2}{*}{ Variáveis } & \multicolumn{2}{|c|}{$\begin{array}{c}\text { Condições } \\
\text { boas de SM }\end{array}$} & \multicolumn{2}{|c|}{$\begin{array}{c}\text { Condições } \\
\text { regulares de SM }\end{array}$} & \multicolumn{2}{|c|}{$\begin{array}{l}\text { Condições } \\
\text { ruins de SM }\end{array}$} & \multirow[t]{2}{*}{$\mathbf{p}$} \\
\hline & $\mathbf{n}$ & $\%$ & $\mathbf{n}$ & $\%$ & $\mathbf{n}$ & $\%$ & \\
\hline \multicolumn{8}{|c|}{ Diminuição de renda } \\
\hline Sim & 17 & 16,67 & 30 & 29,41 & 11 & 10,78 & $0,011^{\star *}$ \\
\hline Não & 26 & 25,49 & 13 & 12,75 & 5 & 4,90 & \\
\hline \multicolumn{8}{|c|}{ Abalar-se ao ver notícias sobre a pandemia } \\
\hline Sim & 26 & 25,49 & 39 & 38,24 & 15 & 14,71 & $0,012^{*}$ \\
\hline Não & 17 & 16,67 & 4 & 3,92 & 1 & 0,98 & \\
\hline \multicolumn{8}{|c|}{ Realização de atividades complementares (ex: yoga, costura) } \\
\hline Sim & 28 & 27,45 & 16 & 15,69 & 1 & 0,98 & $0,001^{*}$ \\
\hline Não & 15 & 14,71 & 27 & 26,47 & 15 & 14,71 & \\
\hline
\end{tabular}

SM: saúde mental *Teste do Qui-quadrado com correção de Yates. **Teste do qui-quadrado

Fonte: Autores (2020).

Em função do período de distanciamento social, 58 (56,86\%) indivíduos relataram que tiveram sua renda afetada. Além disso, alguns dos entrevistados relataram dificuldades na adesão de insumos (frascos de insulina, seringas, materiais para verificação da glicemia, canetas de insulina e medicamentos orais). A Tabela 3 exibe a associação da dificuldade de adesão dos insumos ao déficit de renda $(p<0,000)$ e ao número de verificações da glicemia por dia $(p=0,003)$, assumindo que, com a falta de insumos, o número de verificações diárias diminui. 
Tabela 3 - Influência da diminuição da renda na adesão de insumos e sua consequência no número de verificações diárias da glicemia. Rio de Janeiro, RJ, Brasil, 2020

\begin{tabular}{|c|c|c|c|c|c|}
\hline \multirow[t]{2}{*}{ Variáveis } & \multicolumn{2}{|c|}{$\begin{array}{c}\text { Possuem } \\
\text { dificuldades na } \\
\text { adesão de insumos }\end{array}$} & \multicolumn{2}{|c|}{$\begin{array}{c}\text { Não possuem } \\
\text { dificuldades na } \\
\text { adesão de insumos }\end{array}$} & \multirow[t]{2}{*}{$\mathbf{p}$} \\
\hline & $\mathbf{n}$ & $\%$ & $\mathbf{n}$ & $\%$ & \\
\hline \multicolumn{6}{|c|}{ Diminuição de renda } \\
\hline Sim & 37 & 36,27 & 21 & 20,59 & \multirow[t]{2}{*}{$<0,000^{\star}$} \\
\hline Não & 4 & 3,92 & 40 & 39,22 & \\
\hline \multicolumn{6}{|c|}{ Freq. de verificação da glicemia p/dia } \\
\hline $1 \times$ ao dia & 8 & 7,84 & 13 & 12,76 & \multirow[t]{5}{*}{$0,001 *$} \\
\hline $2 x$ ao dia & 11 & 10,78 & 7 & 6,86 & \\
\hline $3 x$ ao dia & 2 & 1,96 & 6 & 5,88 & \\
\hline $4 x$ ou mais & 14 & 13,73 & 9 & 8,82 & \\
\hline Não verifica & 5 & 4,90 & 27 & 26,47 & \\
\hline
\end{tabular}

*Teste do Qui-quadrado com correção de Yates

Fonte: Autores (2020).

Também foi questionada a regularidade das consultas durante o período de distanciamento social, e os dados mostraram que 35 indivíduos $(34,31 \%)$ relataram interrupção das consultas, $49(48,04 \%)$ disseram se encontrar irregulares e 18 (17,65\%) relataram seguir com regularidade. A respeito dos teleatendimentos, $14(13,7 \%)$ disseram ter consultas via WhatsApp ${ }$, seis $(5,9 \%)$ por vídeo, $10(9,8 \%)$ por ligação telefônica e 72 $(70,6 \%)$ relataram não ter realizado nenhum tipo de teleatendimento no período.

Em relação ao uso de suplementos, 59 (57,8\%) indivíduos relataram, após o início do período de distanciamento social, iniciar o uso de suplementos como suposta forma de proteção, entre eles: suplementos vitamínicos, remédios para aumento da imunidade, chás, vitamina C, Cloridrato de Metformina e Gilbenclamida (acredita-se que descreveu seu tratamento para o DM) e Ivermectina. Relataram não utilizar nenhum tipo de suplemento $43(42,2 \%)$ indivíduos.

\section{DISCUSSÃO}

A amostra analisada neste estudo designa-se correspondente às indicadas em outros estudos, considerando a predominância do sexo feminino, da idade adulta e a prevalência de indivíduos com DM tipo II ${ }^{(11-13)}$.

Foram detectadas mudanças durante o período de distanciamento social, sendo que - peso corporal se apresentou aumentado. Por meio das análises inferenciais, pode-se identificar uma associação entre aumento de peso e alimentação inadequada (32\% referem não ter hábitos alimentares adequados) e déficit na prática de exercícios físicos ( $52 \%$ não realizam tal prática). Estudos ressaltam que, na DM tipo II, o sobrepeso e a obesidade aumentam a incidência e gravidade do SARS-CoV-2 ${ }^{(14-15)}$. 
A obesidade é um fator de risco conhecido para a ventilação anormal e pode contribuir para a redução do resíduo funcional da capacidade pulmonar ${ }^{(16)}$. Além disso, é necessário frisar que pesquisas enfatizam que o aumento de peso, nesse cenário pandêmico, compromete a imunidade dos indivíduos frente à resposta ao vírus, e que o aumento na mortalidade pela covid-19 é maior em países onde a incidência de obesidade é mais proeminente ${ }^{(14)}$. De forma a minimizar o risco, as recomendações durante o distanciamento social são a manutenção da regularidade na dieta diária e dos exercícios físicos em casa ${ }^{(17)}$.

Além das repercussões no âmbito epidemiológico, o novo coronavírus também produz impactos psicológicos, sociais e econômicos que interferem diretamente na vida da população. O alto nível de desemprego associado à recorrência de suspensões de contratos e jornada de trabalho, e a baixa disponibilidade de ofertas trabalhistas em função do baixo desempenho das empresas, provoca a permanência desse quadro ${ }^{(18)}$. $\mathrm{Na}$ amostra analisada, $57 \%$ dos indivíduos demonstraram sofrer consequências a partir do prejuízo socioeconômico decorrente da pandemia, de acordo com a análise realizada, associada com a dificuldade na adesão de insumos para verificação diária da glicemia.

Este achado pode implicar em pior controle da doença, já que a monitoração glicêmica é um dos pilares para um bom controle metabólico e, no período da atual pandemia, vem sendo recomendada a sua realização de forma rigorosa em pessoas com diabetes que ainda não foram infectadas com a covid-19(19).

Em continuidade à questão do controle glicêmico, constatou-se que $32 \%$ não realizam a verificação diária, $21 \%$ somente uma vez ao dia, $18 \%$ duas vezes ao dia e $31 \%$ mais do que três vezes ao dia. Estes achados estão de acordo com outros estudos que retratam a automonitorização da glicemia capilar ${ }^{(20)}$. O padrão de frequência de monitoração glicêmica encontrada nesta amostra sugere uma precarização do controle da doença, já que estudos mostram que a concentração sérica de $\mathrm{HbA1c}$ era inversamente proporcional ao número de dosagens da glicemia capilar por meio do automonitoramento ao dia ${ }^{(21-22)}$.

Mesmo com poucos estudos relacionados ao objeto de pesquisa, dados observados em outras infecções (como SARS-CoV-1 e influenza H1N1) atestaram que pacientes com controle glicêmico deficiente possuem maior incidência de complicações e morte ${ }^{(17)}$. De acordo com a SBD, é imprescindível o respeito à recomendação de acompanhamento à dosagem glicêmica em diferentes momentos da rotina: em jejum, após as refeições e antes de dormir, em uma quantidade que varia de três a seis vezes ao dia, para a atuação imediata corrigindo episódios de hipoglicemia ou picos hiperglicêmicos, evitando possíveis complicações ${ }^{(23)}$.

A exacerbada exposição midiática acerca da crescente porcentagem de infectados e mortos pela covid-19, a rigorosidade do distanciamento social e os prejuízos financeiros são oportunos para o desencadeamento ou agravamento de distúrbios psicológicos, principalmente o transtorno de ansiedade e depressão, não contribuindo para a manutenção da saúde mental e o descuido adicional da fisiológica. Um estudo feito na Etiópia em abril de 2020 indicou um aumento significativo na persistência de sintomas de depressão comparando com os dados anteriores à pandemia ${ }^{(24)}$. No presente estudo, observa-se que um total de $78 \%$ foi abalado mentalmente por conta do enfoque corriqueiro demonstrado em meios de comunicação. Esse fato pode ser maximizado pela falta de suporte profissional, visto que ocorreu na população estudada a suspensão de consultas, algumas sendo substituídas pelo teleatendimento - não inclusivo a toda a população.

Com o objetivo de amenizar o sofrimento psicológico e manejo do estresse para a promoção do bem-estar, $44 \%$ dos indivíduos estudados realizaram atividades complementares como yoga, artesanato, costura etc. A análise indica que existe uma relação positiva entre estas atividades complementares e a melhoria autorrelatada da saúde mental. Tais efeitos vão ao encontro de pesquisas que comprovam resultados benéficos relacionados à realização de práticas como yoga, atuando na melhoria do bem-estar e qualidade de vida, reduzindo sintomas de ansiedade e depressão(25). 
No que tange à ausência de consultas, citada anteriormente, 34,3\% relataram a interrupção total das consultas com profissionais de saúde. Este cenário de suspensão ou irregularidade nas consultas com profissionais de saúde propiciou a ascensão do teleatendimento, "considerado como alternativa eficaz às visitas presenciais de pacientes com outras necessidades de cuidados de saúde, ajudando a preservar os serviços para aqueles que mais necessitam de cuidados pessoais" (26:12). Porém, esta inovação tecnológica adaptada ao período de distanciamento social não atingiu parte dos sujeitos deste estudo, visto que $71 \%$ da amostra não realizaram nenhum tipo de atendimento por meio digital.

Tal distanciamento pessoal ou virtual dos profissionais de saúde favorecem casos de automedicação, já que há uma inquietação dos indivíduos com a melhora da imunidade como forma de prevenção ao vírus circulante. Os dados analisados mostram que, em decorrência desta preocupação, 58\% começaram a usar algum medicamento, destes 27,5\% fizeram uso da vitamina $C$, que apesar das contradições históricas dos pesquisadores, parece exercer um nível potencial de prevenção à pneumonia e evolução de um quadro de piora pela covid-19(27).

Algumas limitações neste estudo são evidenciadas pela dificuldade na obtenção de pesquisas de âmbito nacional, que dificultam a discussão, pois artigos com sujeitos de estudo de culturas distantes da brasileira, como os asiáticos, têm como base uma cultura, costumes, hábitos e características diferentes.

\section{CONSIDERAÇÕES FINAIS}

Ficou evidenciado por meio do estudo que, durante o período de distanciamento social, os portadores de DM apresentaram aumento de peso associado à má alimentação e ao déficit de atividade física. Apresentaram prejuízos econômicos, que refletindo na baixa adesão de insumos que auxiliam o tratamento do DM, o que prejudicou a monitoração glicêmica. O estado de saúde mental também foi impactado negativamente, especificamente pelas notícias da pandemia veiculadas nos meios de comunicação. $O$ distanciamento social influenciou as consultas profissionais, tornando-as irregulares ou ausentes. Nesta amostra, o teleatendimento não se mostrou frequente.

A pandemia de covid-19 representa uma adversidade sem precedentes para os pacientes com DM. Mesmo com a diminuição dos casos em alguns países, o distanciamento social para a parcela da população que compõe grupo de risco - como esses pacientes ainda será uma realidade por tempo indeterminado.

Conhecer as características desta população, considerada grupo de risco para o covid-19, como se propôs o presente estudo, é importante para o desenvolvimento de práticas de educação em saúde mais otimizadas e específicas. Para enfrentar os problemas encontrados pelos indivíduos com DM, a equipe multiprofissional que os assiste deve focar sua atenção em fatores que estão influenciando negativamente o controle metabólico, como aumento de peso e monitoração glicêmica deficitária.

Vale ressaltar que a implantação de estratégias de atendimento a distância pode ser uma solução, neste momento, para que as pessoas com DM continuem a ter um apoio profissional para as demandas físicas e mentais que surgem em meio à pandemia. 
1. Zhu N, Zhang D, Wang W, Li X, Yang B, Song J, et al. A Novel Coronavirus from patients with pneumonia in China, 2019. N. Engl. J. Med. [Internet]. 2020 [acesso em 30 jul 2020]; 382(8). Disponível em: http://dx.doi.org/10.1056/nejmoa2001017.

2. Wang D, Hu B, Hu C, Zhu F, Liu X, Zhang J, et al. Clinical characteristics of 138 hospitalized patients with 2019 novel coronavirus-infected pneumonia in Wuhan, China. JAMA. [Internet]. 2020 [acesso em 30 jul 2020]; 323(11). Disponível em: https://doi.org/10.1001/jama.2020.1585.

3. Zhou F, Yu T, Du R, Fan G, Liu Y, Liu Z, et al. Clinical course and risk factors for mortality of adult inpatients with COVID-19 in Wuhan, China: a retrospective cohort study. Lancet. [Internet]. 2020 [acesso em 30 jul 2020]; 395(10229). Disponível em: https://doi.org/10.1016/S0140-6736(20)30566-3.

4. Wu Z, McGoogan JM. Characteristics of and important lessons from the Coronavirus disease 2019 (COVID-19) Outbreak in China. JAMA [Internet]. 2020 [acesso em 30 jul 2020]; 323(13). Disponível em: http://dx.doi.org/10.1001/jama.2020.2648.

5. Wang D, Hu B, Hu C, Zhu F, Liu X, Zhang J, et al. Clinical characteristics of 138 hospitalized patients with 2019 novel Coronavirus-Infected pneumonia in wuhan, China. JAMA. [Internet]. 2020 [acesso em 30 jul 2020]; 323(11). Disponível em: http://doi.org/10.1001/jama.2020.1585.

6. Wu C, Chen X, Cai Y, Xia J, Zhou X, Xu S, et al. Risk factors associated with acute respiratory distress syndrome and death in patients with coronavirus disease 2019 pneumonia in Wuhan, China. JAMA Intern. Med. [Internet]. 2020 [acesso em 30 jul 2020]; 180(7). Disponível em: http://doi.org/10.1001/ jamainternmed.2020.0994.

7. Guan W, Ni Z, Hu Y, Liang W, Ou C, He J, et al. Clinical characteristics of coronavirus disease 2019 in China. N Engl J Med. [Internet]. 2020 [acesso em 30 jul 2020]; 382. Disponível em: http://doi.org/10.1056/ NEJMoa2002032.

8. Zhang JJ, Dong X, Cao YY, Yi-bin Y, You-qin Y, Cezmi A, et al. Clinical characteristics of 140 patients infected bySARS-CoV-2 in Wuhan, China. Allergy. [Internet]. 2020 [acesso em 30 jul 2020]; 75(7). Disponível em: https://pubmed.ncbi.nlm.nih.gov/32077115/\#: :text=Fever\%20(91.7\%25)\%2C\%20 cough\%20(,self\%2Dreported\%20by\%20several\%20patients.

9. Sociedade Brasileira de Diabetes (SBD). COVID-19 e diabetes. [Internet]. SBD; 2020 [acesso em 21 jul 2020]. Disponível em: https://www.diabetes.org.br/covid-19/covid-19-e-diabetes/.

10. Michels MJ, Coral MHC, Sakae TM, Damas TB, Furlanetto LM. Questionário de atividades de autocuidado com o diabetes: tradução, adaptação e avaliação das propriedades psicométricas. Arq Bras Endocrinol Metab. [Internet]. 2010 [acesso em 10 mar 2020]; 54(7). Disponível em: http://doi.org/10.1590/ S0004-27302010000700009.

11. Batista JMF, Becker TAC, Zanetti ML, Teixeira CR de S. O ensino em grupo do processo de aplicação de insulina. Rev Eletr Enf. [Internet]. 2013 [acesso em 30 jul 2020]; 15(1). Disponível em: http://doi. org/10.5216/ree.v15i1.16179.

12. Diógenes MAR, Souza AKP de, Cavalcante IP, Lopes LCO, Rebello MMC. Insulinoterapia: conhecimento e práticas utilizadas por portadores de diabetes mellitus tipo 2. Rev. enferm. UERJ. [Internet]. 2012 [acesso em 30 jul 2020]; 20(6). Disponível em: https://www.e-publicacoes.uerj.br/index. php/enfermagemuerj/article/view/5998.

13. Oliveira ABAS, Silva LF, Mello SDP, Ferreira MS, Silva JCS. Conhecimento de portadores de diabetes mellitus acerca da conservação da insulina. Rev. Saúde (Sta. Maria). [Internet]. 2019 [acesso em 30 jul 2020]; 45(2). Disponível em: http://doi.org/10.5902/2236583437342.

14. Dietz W, Santos-Burgoa C. Obesity and its Implications for COVID-19 Mortality. Obesity. [Internet]. 2020 [acesso em 30 jul 2020]; 28(6). Disponível em: http://doi.org/10.1002/oby.22818.

15. Hussain A, Mahawar K, Xia Z, Yang W, El-Hasani S. Obesity and mortality of COVID-19. Metaanalysis. Obes Res Clin Pract. [Internet]. 2020 [acesso em 30 jul 2020]; 14(4). Disponível em: https://doi. org/10.1016/j.orcp.2020.07.002. 
16. Selvin, E, Juraschek SP. Diabetes epidemiology in the COVID-19 Pandemic. Diabetes Care. [Internet]. 2020 [acesso em 30 jul 2020]; 43(8). Disponível em: https://doi.org/10.2337/dc20-1295.

17. Singh AK, Gupta R, Ghosh A, Misra A. Diabetes in COVID-19: prevalence, pathophysiology, prognosis and practical considerations. Diabetes Metab Syndr. [Internet]. 2020 [acesso em 30 jul 2020]; 14(4).

Disponível em: http://doi.org/10.1016/j.dsx.2020.04.004.

18. Fundação Oswaldo Cruz (FIOCRUZ). Impactos sociais, econômicos, culturais e políticos da pandemia. [Internet]. FIOCRUZ; 2020 [acesso em 21 jul 2020]. Disponível em: https://portal.fiocruz.br/impactossociais-economicos-culturais-e-politicos-da-pandemia.

19. Bornstein SR, Rubino F, Khunti K, Mingrone G, Hopkins D, Birkenfeld AL, et al. Practical recommendations for the management of diabetes in patients with COVID-19. Lancet. [Internet]. 2020 [acesso em 30 jul 2020]; 8(6). Disponível em: http://doi.org/10.1016/S2213-8587(20)30152-2.

20. Teixeira CR de S, Zanetti ML, Landim CAP, Becker TAC, Santos ECB dos, Franco RC, et al. Automonitorização da glicemia capilar no domićlio: revisão integrativa da literatura. Rev Eletr Enf. [Internet]. 2009 [acesso em 30 jul 2020]; 11(4). Disponível em: http://deploy.extras.ufg.br/projetos/fen revista/v11/n4/pdf/v11n4a27.pdf.

21. Ziegler R, Heidtmann B, Hilgard D, Hofer S, Rosenbauer J, Holl R, et al. Frequency of SMBG correlates with $\mathrm{HbA} 1 \mathrm{c}$ and acute complications in children and adolescents with type 1 diabetes. Pediatr diabetes. [Internet]. 2011 [acesso em 30 jul 2020]; 12(1). Disponível em: http://doi.org/10.1111/j.13995448.2010.00650.x.

22. Miller KM, Beck RW, Bergenstal RM, Goland RS, Haller MJ, McGill JB, et al. Evidence of a strong association between frequency of self-monitoring of blood glucose and hemoglobin A1c levels in T1D exchange clinic registry participants. Diabetes Care. [Internet]. 2013 [acesso em 30 jul 2020]; 36(7). Disponível em: http://doi.org/10.2337/dc12-1770.

23. Sociedade Brasileira de Diabetes (SBD). Diretrizes da Sociedade Brasileira de Diabetes: 2019-2020/ Sociedade Brasileira de Diabetes. São Paulo: Clannad; 2019.

24. Organização Mundial da Saúde (OMS). O impacto da pandemia na saúde mental das pessoas já é extremamente preocupante. [Internet]. OMS; 2020 [acesso em 21 jul 2020]. Disponível em: https://brasil. un.org/pt-br/85787-oms-o-impacto-da-pandemia-na-saude-mental-das-pessoas-ja-e-extremamentepreocupante.

25. Rosa AL da, Miranda AV de S. O yoga como prática integrativa na promoção de saúde mental: uma ferramenta ética para o cuidado de si. Rizoma. [Internet]. 2017 [acesso em 30 jul 2020]; 1(2). Disponível em: http://periodicos.estacio.br/index.php/rizoma/article/viewFile/3180/alvaro.

26. Caetano R, Silva AB, Guedes ACCM, Paiva CCN de, Ribeiro G da R, Santos DL, et al. Desafios e oportunidades para telessaúde em tempos da pandemia pela COVID-19: uma reflexão sobre os espaços e iniciativas no contexto brasileiro. Cad. Saúde Pública. [Internet]. 2020 [acesso em 30 jul 2020]; 36(5). Disponível em: http://doi.org/10.1590/0102-11x00088920.

27. Gupta R, Ghosh A, Singh AK, Misra A. Clinical considerations for patients with diabetes in times of COVID-19 epidemic. Diabetes Metab Syndr. [Internet]. 2020 [acesso em 30 jul 2020]; 14(3). Disponível em: http://doi.org/10.1016/j.dsx.2020.03.002. 
COMO REFERENCIAR ESTE ARTIGO:

Pedroza GG de O, Monção AC de M, Valladares H de O, Mello SD de P, VH de MP de, Silva JCS da, et al. Hábitos de vida de pessoas com diabetes mellitus durante a pandemia de covid-19. Cogitare enferm. [Internet]. 2021 [acesso em "colocar data de acesso, dia, mês abreviado e ano"]; 26. Disponível em: http://dx.doi.org/10.5380/ ce.v26i0.75769.

Recebido em: 08/08/2020

Aprovado em: 30/11/2020

Editora associada: Luciana Puchalski Kalinke

Autor Correspondente:

Marcela dos Santos Ferreira

Centro Federal de Educação Tecnológica Celso Suckow da Fonseca - Nova Iguaçu, RJ, Brasil

Email: cceccella@hotmail.com

Contribuição dos autores:

Contribuições substanciais para a concepção ou desenho do estudo; ou a aquisição, análise ou interpretação de dados do estudo - GGOP, ACMM, HOV, SDPM, VHMPS, JCSS, MSF

Elaboração e revisão crítica do conteúdo intelectual do estudo - GGOP, ACMM, HOV, SDPM, VHMPS, JCSS, MSF Aprovação da versão final do estudo a ser publicado - GGOP, ACMM, HOV, SDPM, VHMPS, JCSS, MSF

Responsável por todos os aspectos do estudo, assegurando as questões de precisão ou integridade de qualquer parte do estudo - GGOP, ACMM, HOV, SDPM, VHMPS, JCSS, MSF

Copyright $\odot 2021$ Este é um artigo em acesso aberto distribuído nos termos da Licença Creative Commons Atribuição, que permite o uso irrestrito, a distribuição e reprodução em qualquer meio desde que o artigo original seja devidamente citado. 